\title{
CIRUGÍA TRANS-ORAL ROBÓTICA PARA MANEJO DE CÁNCER DE OROFARINGE*
}

\author{
Drs. Arturo Madrid M. ${ }^{1}$, Felipe Capdeville F. ${ }^{1}$, Luis Felipe Zanolli de S. ${ }^{1}$, \\ Marcelo Orvieto S. ${ }^{1}$, Hugo Rojas P. ${ }^{1}$, Felipe Panussis F. ${ }^{1}$, Fabio Valdés G. ${ }^{1}$ \\ 1 Departamento de Cirugía y Oncología Clínica Alemana, Santiago. \\ Chile.
}

\begin{abstract}
Trans-oral robotic surgery for tongue cancer

Background: The traditional treatment of oropharyngeal cancer was based mainly in radio and chemotherapy, aiming to avoid organ excisions. However, the adverse effects of these therapies prompted the development of new therapies. Among these, trans-oral robotic surgery (TORS) has promising results. Case reports: We report two patients operated using this technique. An 82 years old woman treated for a tumor in the base of the tongue six years ago with radiotherapy. A new lesion in the tongue was found and excised by TORS uneventfully. The pathology report confirmed the complete excision of the malignant lesion. A 57 years old male with a history of palate cancer treated with radiotherapy in 1990. In 2013 a submandibular mass was biopsied confirming the presence of a squamous carcinoma metastasis. During a bilateral suprahyoid dissection, a tumor in the base of the tongue was found. In a second intervention the tumor was excised by TORS. The pathology reports tumor free borders but near the lesion. Postoperative radiotherapy was recommended.

Key words: Oropharyngeal cancer, tongue, trans-oral surgery.
\end{abstract}

\section{Resumen}

Introducción: El cáncer de orofaringe representa una importante causa de morbimortalidad en la esfera de las patologías de cabeza y cuello en la actualidad. Su tratamiento tradicional por mucho tiempo consideró la conservación de órganos, utilizando mayoritariamente la radio y quimioterapia como herramientas terapéuticas. Los efectos no deseados tanto de las terapias médicas como las quirúrgicas han obligado a desarrollar técnicas nuevas para el manejo de estas enfermedades. Es así que a partir del año 2006 se ha ido desarrollando a nivel mundial la técnica de cirugía robótica transoral (TORS). La morbilidad y resultados oncológicos de este procedimiento hasta ahora se ven alentadores, por lo que se está transformando en una alternativa terapéutica que debemos manejar. Casos clínicos: Se presentan los dos primeros casos a nivel país realizados en esta institución de cánceres de orofaringe tratados con la técnica tradicional y después de largo tiempo recidivados. Se les realizó cirugía resectiva de sus tumores a través de TORS; se describen los resultados de estos procedimientos.

Palabras clave: Cáncer orofaringe, cirugía robótica, cirugía transoral.

*Recibido el 10 de junio de 2014 y aceptado para publicación el 12 de septiembre de 2014.

Conflicto de Interés: los autores declaran no tener conflicto de interés con la confección de este artículo.

Correspondencia: Dr. Arturo Madrid M.

amadrid@alemana.cl 


\section{Introducción}

A nivel mundial el cáncer de orofaringe representa el 3\% de todos los cánceres, con una mortalidad de 8,3 por 100.000 habitantes $^{1}$. En Chile tiene una incidencia en hombres de 3,3 por 100.000 habitantes y 1,3 en el caso de las mujeres, presentando una tasa de mortalidad de 1,9 y 0,6 por 100.000 en hombres y mujeres respectivamente, según datos estimados del Ministerio de Salud ${ }^{2}$. La variedad histológica más frecuente es el carcinoma espinocelular, en el 95\% de los $\operatorname{casos}^{1}$. Como factores etiológicos más importantes en nuestro medio se encuentra el consumo de tabaco, alcohol y la infección por virus papiloma humano (HPV), sobretodo con serotipos de alto riesgo como el 16 y $18^{3,4}$. Tradicionalmente el tratamiento de estos tumores se realiza con la radioterapia exclusiva, terapias combinadas como la radioquimioterapia y/o la resección quirúrgica, siendo esta última la menos frecuente como indicación por su importante morbilidad asociada. En el último tiempo la cirugía mínimamente invasiva se ha posicionado como una opción efectiva para el tratamiento de estos tumores, permitiendo disminuir los efectos colaterales no deseados que se producen con las terapias estándares ${ }^{5,6}$. En este contexto, la Cirugia Trans-Oral Láser, y por otro parte, la Cirugía Trans-Oral Robótica o TORS (Trans-Oral Robotic Surgery) han ganado mucho terreno en los últimos años para el tratamiento de esta patología oncológica.

TORS fue introducida por primera vez en la literatura por Weinstein en el año 20067. Desde esta publicación se ha ido desarrollando el uso de TORS en el manejo de patología maligna en cabeza y cuello, en especial de laringe y orofaringe. Tiene resultados oncológicos comparables, como también funcionales con menor compromiso de la vía aérea (posibilidad de no utilizar traqueostomía) y menor trastorno de deglución ${ }^{5-8}$.

Se presentan dos casos clínicos de cáncer de orofaringe tratados con TORS en el equipo de cabeza y cuello de nuestra institución, previa autorización con consentimiento informado.

\section{Caso clínico 1}

Se describe una paciente de género femenino con antecedentes de tabaquismo crónico 25 paquetes/año. El año 2008 (con 78 años de edad) es estudiada por un trastorno de deglución, donde se diagnostica un cáncer de base de lengua. Completa su estudio con una etapificación cT1N0M0, siendo evaluada en el comité de cabeza y cuello recomendándose radioterapia exclusiva. Recibe 66Gy en orofaringe, y 50Gy a linfonodos cervicales bilaterales. Evoluciona con una adecuada tolerancia, con leve odinofagia, radiodermitis asociada a xerostomía y disfagia leve. Se mantiene en controles hasta que en octubre de 2013 en una nasofibrolaringoscopia, se pesquisa una lesión solevantada con una ulceración de aproximadamente $5 \mathrm{a} 8 \mathrm{~mm}$ en la base de lengua hacia la derecha. Se estudia con PET- CT (Figura 1) que muestra probable recidiva en el sitio del tumor primario sin metástasis a distancia. Se realiza biopsia, que confirma un carcinoma espinocelular, siendo evaluada en el comité de cabeza y cuello. En consideración a sus 82 años, con un territorio irradiado, y teniendo una lesión de pequeño tamaño, se sugiere TORS para evitar la morbilidad de una cirugía abierta de rescate. Esta se realiza en diciembre de 2013. Previa traqueostomía para protección de la vía aérea se utiliza un abreboca autoestático de aro. Se implementa el Robot Da Vinci ${ }^{\circledR}$ (Intuitive Surgical, Sunnyvale, CA, USA). Luego de la instalación de los brazos robóticos se comprueba la visión completa del tumor. Se utiliza ópticas de $0^{\circ}$ y de $30^{\circ}$, además de una pinza y tijeras monopolar en los brazos del robot (Figura 2). Se logra resecar tumor con márgenes libres confirmados con biopsia rápida. Cirugía sin incidentes y sin sangrado importante. Paciente se envía a UCI siendo desconectada de ventilación mecánica al segundo día. Deglución normal y retiro de traqueostomía al $4^{\circ}$ y $5^{\circ}$ día postoperatorios, respectivamente. Deglutiendo normal al $4^{\circ}$ día post operatorio. La biopsia diferida confirma carcinoma espinocelular con márgenes negativos, por lo que el comité sugiere sólo observación.

\section{Caso clínico 2}

Paciente hombre de 57 años, con antecedentes de resistencia a la insulina, alcoholismo, tabaquismo por 20 años (30 paq/años) suspendido. Tiene historia de un cáncer de paladar blando y úvula, tratado en 1990 con radioterapia exclusiva al sitio del primario y cuello bilateral. En julio de 2013, por aumento de volumen submandibular se realiza biopsia que informa metástasis de carcinoma escamoso. Es sometido a un vaciamiento supraomohioídeo bilateral, además de un examen bajo anestesia de la vía aérea superior, que mostró una lesión ulcerada en base de lengua cuya biopsia diferida confirma la presencia de un segundo primario de orofaringe, al cual no se le realizó tratamiento. Se estudia con PET-CT que confirma un tumor en base de lengua además de adenopatías en grupo IA y IB. Consulta con estos antecedentes, siendo evaluado en el comité de cabeza y cuello sugiriéndose TORS y redisección cervical. En pabellón, posterior a traqueostomía, se implementa el Robot Da Vinci® (Intuitive Surgical, 


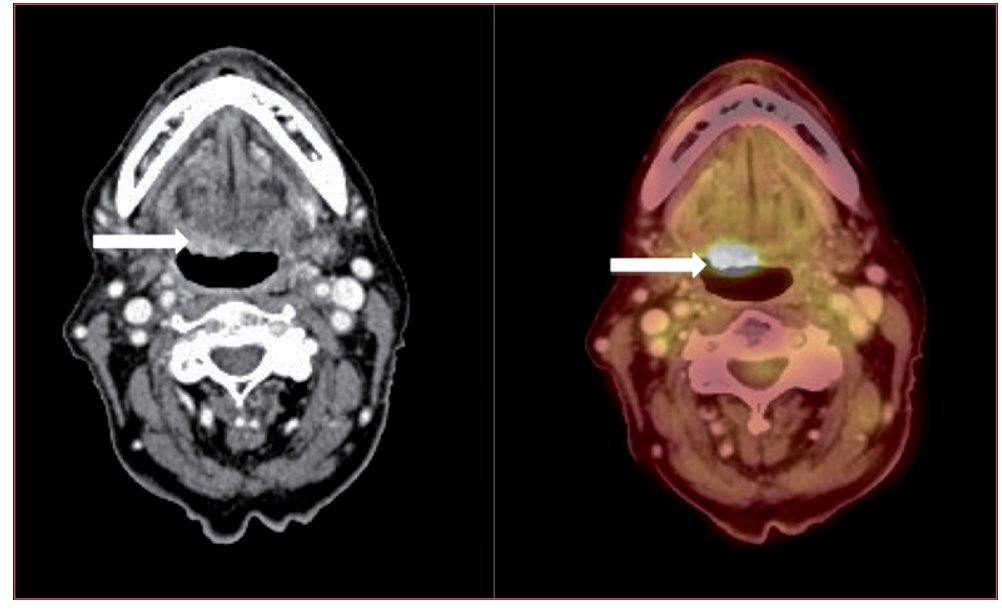

Figura 1. Imagen de PET CT que muestra zona de recidiva 5 años después del primario de base de lengua.

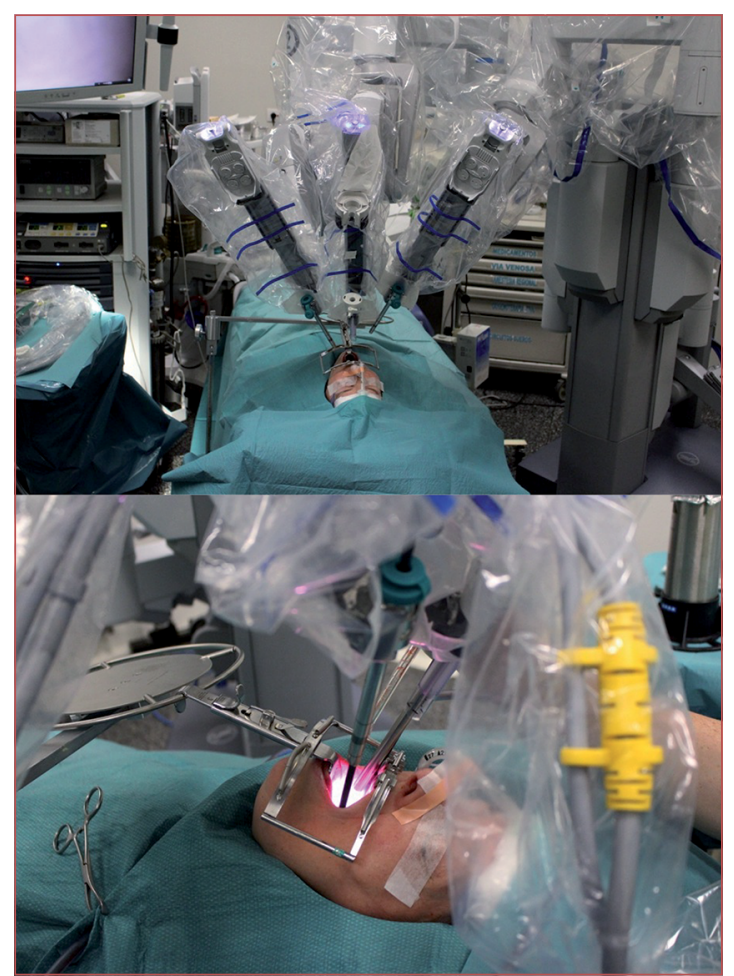

Figura 2. Posicionamiento del Robot Da Vinci ${ }^{\circledR}$ (Intuitive Surgical, Sunnyvale, CA, USA) para cirugía transoral.

Sunnyvale, CA, USA). Luego de la instalación de los brazos robóticos se comprueba la visión completa del tumor, lográndolo resecar con márgenes libres confirmados con biopsia rápida. Evoluciona en forma favorable, con ejercicios de deglución al sexto día. La traqueotomía se retira al $8^{\circ}$ día. Biopsia diferida informa carcinoma epidermoide con diáme- tro de 2,1 cm, con márgenes libres pero cercanos. La evaluación por comité postcirugía recomienda radioterapia postoperatoria.

\section{Discusión}

El tratamiento cáncer de orofaringe ha evolucionado en las últimas décadas 9 . En la actualidad el tratamiento con radioterapia o quimioradioterapia ha sido el de elección en desmedro de la cirugía abierta, esto es principalmente porque los procedimientos quirúrgicos tradicionales presentan un gran número de complicaciones las que se ven incrementadas especialmente en los territorios que requerirán o fueron sometidos a radioterapia ${ }^{10,11}$.

Dentro de las complicaciones más frecuentes encontradas en el tratamiento de estos pacientes se puede considerar la xerostomía, dermatitis actínica, trastorno de la deglución, uso de traqueotomía y gastrostomía en forma prolongada, osteoradionecrosis, mucositis y neutropenias, las cuales disminuyen la calidad de vida de manera considerable, especialmente en los pacientes jóvenes. En este sentido, TORS es una herramienta que permite buen control oncológico como también buenos resultados funcionales, reduciendo los efectos no deseados de los tratamientos estándares actuales; incluso se estudian hoy tratamientos con menores dosis de radioquimioterapia postoperatoria en estos pacientes ${ }^{12-14}$.

TORS permite preservar mayor tejido normal como también su irrigación e inervación permitiendo una cicatrización óptima y evita dañar innecesariamente los tejidos sanos adyacentes al tumor, reduciendo los extensos abordajes que son necesarios para una resección oncológicamente satisfactoria.

La cirugía robótica en nuestro país se ha introdu- 
cido rápidamente en los últimos años especialmente en el área de la urología y ginecología ${ }^{15}$. El desarrollo en el área de cabeza y cuello a nivel mundial aún está en constante evaluación, siendo tema esencial de los últimos congresos internacionales. Nosotros consideramos que la indicación para este procedimiento debe ser muy precisa, en casos muy especiales y evaluados previamente por un comité multidisciplinario. La experiencia presentada es pionera en nuestro país con el uso de cirugía robótica en el manejo de esta patología. Esperamos con el tiempo contar con mayor experiencia en el manejo avanzado de esta patología con estas nuevas herramientas, que nos permitirán disminuir los riesgos asociados a los tratamientos convencionales en estos pacientes.

\section{Referencias}

1. Guzmán P. Carcinoma epidermoide oral y orofaríngeo. Estudio clínico-patológico. Rev Chil Cir. 2011;63:2506.

2. Primer informe de registros poblacionales de cáncer en Chile. Quinquenio 2003-2007. Ministerio de Salud de Chile, 2012.

3. Dowthwaite SA, Franklin JH, Palma DA, Fung K, Yoo J, Nichols AC. The role of transoral robotic surgery in the management of oropharyngeal cancer: a review of the literature. ISRN Oncol. 2012;2012:945162.

4. Moore EJ, Olsen SM, Laborde RR, García JJ, Walsh FJ, Price DL, et al. Long-term functional and oncologic results of transoral robotic surgery for oropharyngeal squamous cell carcinoma. Mayo Clin Proc. 2012;87:219-25.

5. Nichols AC, Yoo J, Hammond JA, Fung K, Winquist E, Read N, et al. Early-stage squamous cell carcinoma of the oropharynx: radiotherapy vs trans-oral robotic surgery (ORATOR)-study protocol for a randomized phase II trial. BMC Cancer 2013;13:133.

6. Rinaldi V, Pagani D, Torretta S, Pignataro L. Transoral robotic surgery in the management of head and neck tumours. Ecancermedicalscience 2013;7:359.

7. Genden EM, O'Malley BW, Weinstein GS, Stucken CL, Selber JC, Rinaldo A, et al. Transoral robotic surgery: role in the management of upper aerodigestive tract tumors. Head Neck 2012;34:886-93.

8. O'Malley BW, Weinstein GS, Snyder W, Hockstein NG. Transoral Robotic Surgery (TORS) for Base of Tongue Neoplasms. The Laryngoscope 2006;116:1465-72.

9. Glenny AM, Furness S, Worthington HV, Conway DI, Oliver R, Clarkson JE, et al, The CSROC Expert Panel. Interventions for the treatment of oral cavity and oropharyngeal cancer: radiotherapy. Cochrane Database of Systematic Reviews 2010, Issue 12. Art. $\mathrm{N}^{\circ}$ : CD006387. DOI: 10.1002/14651858.CD006387.pub2

10. Langendijk JA, Doornaert P, Verdonck-de Leeuw IM, Leemans CR, Aaronson NK, Slotman BJ. Impact of late treatment-related toxicity on quality of life among patients with head and neck cancer treated with radiotherapy. Journal of Clinical Oncology 2008;26:3770-6.

11. Benson E, Li R, Eisele D, Fakhry C. The clinical impact of HPV tumor status upon head and neck squamous cell carcinomas. Oral Oncology 2014;50:565-74.

12. Nichols AC, Fung K, Chapeskie C, Dowthwaite SA, Basmaji J, Dhaliwal S, et al. Development of a transoral robotic surgery program in Canada. J Otolaryngol Head Neck Surg. 2013;42:8. doi: 10.1186/1916-0216-42-8.

13. Shah JP, Gil Z. Current concepts in management of oral cancer-surgery. Oral Oncology 2009;45:394-401.

14. Quon H, Richmon JD. Treatment deintensification strategies for HPV-associated head and neck carcinomas. Otolaryngologic Clinics of North America 2012;45:84561.

15. Castillo OA, Vidal I. Cirugía robótica. Rev Chil Cir. [online]. 2012, vol. 64, $\mathrm{n}^{\circ} 1$ [citado 2014-04-25], pp. 88-91. Disponible en: <http://www.scielo.cl/ scielo.php?script $=$ sci_arttext\&pid $=$ S0718-40262012 000100016\&lng=es\&nrm=iso $>$. ISSN 0718-4026. http://dx.doi.org/10.4067/S0718-40262012000100016. 